\title{
Scanning Tunneling Microscopes
}

Commercialisation of scanning tunneling microscopes (STMs) in the pioneering years from about 1986 saw fairly widespread dissemination of some 400 or more units, where the microscope head was supplied either as a table-top device or as a stage that could be bolted on to an existing vacuum system. Digital Instruments, Inc., one of the several manufacturers among those supplying, or about to supply, the European market (see below) also offers a "standalone" head that is designed to be placed on large specimens such as magnetic discs and mirrors. The availability of these various STM stages and their associated control electronics, together with computer interfaces and software, allowed researchers to familiarise themselves with the scientific capabilities of the technique for a relatively small initial outlay (about 150 k SFR).

The combination of excellent scientific results, greater appreciation of potential applications and the improved availability of commercial instruments has wetted appetites. Manufacturers will be placing greater emphasis on the needs of technologically orientated users interested in micrometrology for instance, and of non-traditional users who are not solid state physicists, particularly biologists, biochemists and polymer scientists wishing to exploit atomic force microscopy (AFM). An advantage of AFM is that non-conducting specimens can be imaged in both air and solution to give easily interpreted topographic information. For example, observations of nucleic acids such as DNA tell much about aspects of gene regulation that depend on molecular structures at the 0.02 to $0.25 \mathrm{~nm}$ level, coinciding with the resolutions of most STMs and AFMs.

Specialists in surface science have in the meantime been building for themselves sophisticated, high resolution systems capable of imaging specimens, cleaned in-situ, at very low pressures. Our article by members of a group working at the University of Basel describes one such system. Several companies, notably Omicron Vakuumphysik $\mathrm{GmbH}$ and VG Microscopes Ltd. in Europe, have commercialised complete UHVSTM systems of this type, designed and engineered to production standards. In spite of their relatively high cost (around 300 $350 \mathrm{k}$ SFR on average) and limited sales, interest remains since surface scientists will continue to require these ready-to-use instruments incorporating a full range of ancillary techniques such as low energy electron diffraction, etc.

Some impressive results have been reported recently for state-of-the-art UHVSTMs. The Basel group has been able to image the atomic structures of steps, several atoms high, arising on the surface of silicon crystals (Europhys. Lett., to be published in May 1990). A group based at the University of Aix-Marseilles II, which is collaborating with Micro-Contrôle S.A., have resolved atomic features on the surfaces of small gold clusters (1.0-1.5 nm diameter) deposited in-situ on graphite (Europhys. Lett. 10 (1989) 533). Such clusters have up to now been thought to be too instable to allow imaging at this level of resolution. Other recent results are reviewed in the article by members of a group at Aarhus University who collaborate with Struers in Denmark.

AFMs are presently been supplied by Digital Instruments and by Park Scientific Instruments as a separate microscope stage that can be plugged in to the companies' table-top and bolt-on STM systems. Microscope heads exploiting other imaging techniques will become more readily available, allowing users to continuously upgrade their systems.

There are also commercial development projects underway in Europe and the US aimed at extending the range of AFMs to include UHV versions, either as a bolt-on stage or as an entire UHV system, for high resolution surface science work. The main technical difficulty is to design a head that can support the high temperatures (above about $150{ }^{\circ} \mathrm{C}$ ) encountered during baking to clean the chamber.

\section{What to look for}

The performance of a STM is usually quoted in terms of the lateral and vertical resolutions under the specified operating conditions, which largely depend on the type of microscope stage (table top, bolt on, low temperature, etc.). The design and performance of the stage are crucial, with most manufacturers offering alternative solutions. Important parameters describe:

- positioning of the microscope tip with respect to the specimen: range and speed of the coarse and fine vertical approaches, range and stability of horizontal positioning, viewing of the specimen loptical microscope, video camera, scanning electron mi croscope under development by Omicron): - tip scanning: design of the piezoelectric scanner, its resonant frequency, vertical range, minimum and maximum horizontal ranges, scanning speed, minimum and maximum step sizes, interchangability;

- tips: type, how they are changed, the number that can be parked, possibilities for in-situ cleaning;

- sample: the size that can be mounted, the number parked, possibilities for in-situ cleaning and sample preparation, accessibility of the specimen during STM, interchangability of the sample mount;

The performance of the controller for the microscope head is equally important. Parameters that are often specified include the ranges and settings of the specimen bias voltage and the tunneling current, the scan rate, and the sensitivity and performance (noise, ripple, bandwidth, etc.) of the amplifier for the tunneling current and of the power supply for the scanner. WA Technology's amplifier is highly reputed and, together with Omicron's, is one of the few to be specified in detail.
A personal or micro computer or a workstation is usually interfaced to the controller. The degree of computer control and the power of the computer software largely determine a STM's flexibility and ease of operation. Menu-driven software (WA Technology, Struers) is a great help. Full digital control of the scanning tip is an advantage as it allows imaging under adjustable conditions in addition to the conventional constant current and constant tip separation modes (Digital Instruments). It may also enhance scanning tunneling spectroscopy (STS) capabilities where variations of the current are analysed (fixed point spectroscopy is another option).

Some STMs offer automatic positioning of the tip according to preset values of the tunneling conditions: the time to obtain an image may be specified. Various display formats are available, but the degree of image processing in real-time for edge enhancement, filtering, colour-scale adjustment, calibration, etc. varies significantly between instruments. Most STMs, however, offer image rotation and zooming, corrections for specimen tilt and offset, and the calculation of topographic parameters such as the mean surface roughness.

Some might well envy those fortunate enough to be able to get their hands on one of the very powerful table-top or bolt-on STMs for atom level imaging and spectroscopy that are now available off-the-shelf from several sources.

\section{Suppliers in Europe}

Digital Instruments, Inc.,

6780 Cortona Drive, Santa Barbara,

CA 93117, USA

$\mathrm{TI}++1$ (805) $9688116 / \mathrm{Fax}++1$ (805) 9686627

LK Technologies, Inc.,

3910 Roll Ave., Bloomington,

IN 47401, USA

$\mathrm{TI}++1$ (812) $3324449 / \mathrm{Fax}++1$ (812) 3324493

Micro-Contrôle S.A.,

P.A. de St. Guénault, Rue Jean Mermoz, B.P. 144, F-91005 Every Cedex, France. $\mathrm{TI}++33$ (1) $64979898 / \mathrm{Fax}++33$ (1) 60794561

Omicron Vakuumphysik GmbH,

Idsteiner Str. 78, D-6204 Tanusstein 4. $\mathrm{TI}++49$ (6128) 73044 / Fax ++49 (6128) 73596

\section{Park Scientific Instruments}

476 Ellis St., Mountain View,

CA 94043, USA

$\mathrm{TI}++1$ (415) $9652976 / \mathrm{Fax}++1$ (415) 9652946

Quanscan, Inc.,

959 E. Walnut St., No. 110, Pasadena, CA 91106, USA.

$\mathrm{TI}++1$ (818) $7951163 / \mathrm{Fax}++1$ (818) 4492641

\section{Struers,}

Valhøjs Allé 176, DK-2610 Rødovre Copenhagen, Denmark.

$\mathrm{TI}++45$ (31) $708090 / \mathrm{Fax}++45$ (31) 416544

VG Microscopes Ltd.,

Imberhome Lane, East Grinstead,

West Sussex RH19 1UB, UK.

$\mathrm{TI}++44$ (342) $327211 / \mathrm{Fax}++44$ (342) 315074

WA Technology Ltd.,

Chesterton Mills, French's Road,

Cambridge CB4 3NP, UK.

$\mathrm{TI}++44$ (223) $64714 / \mathrm{Fax}++44$ (223) 460861

European distributors: contact the suppliers. 